\title{
La migration des agriculteurs français outre Atlantique : fait anecdotique ou fait socio-économique?
}

Jean-paul Charvet, Violette Rey

\section{Résumé}

Cet article cerne le profil d'une nouvelle migration d'agriculteurs qui s'est amorcée en direction de l'Amérique à partir des années 1973-75. Ne touchant que de faibles effectifs, peu organisé, sans institution de soutien, le mouvement est difficile à connaître et n'est pas spécifique aux agriculteurs français ; il recherche des terres disponibles et des structures économiques agricoles plus libérales que celles de la France et du Marché Commun.

Les candidats au départ sont originaires des régions de grande culture (Bassin Parisien, Aquitaine). Trois traits caractérisent leur projet : il correspond plus à une attraction du lieu d'arrivée qu'à un refoulement du lieu de départ. II est indissociable d'un projet d'achat de terres ; il ne concerne pas les «paysans pauvres ». Le Canada et l'Argentine sont les premiers pays d'accueil ; la réussite y reste incertaine et difficile parce que les installations ont lieu sur des terres marginales à hauts risques et dans des régions mal desservies.

S'efforçant de mettre à profit le décalage des prix fonciers entre l'Europe et l'Amérique, ce mouvement peut sous certaines conditions intégrer de nouveaux espaces à l'agriculture.

\section{Citer ce document / Cite this document :}

Charvet Jean-paul, Rey Violette. La migration des agriculteurs français outre Atlantique : fait anecdotique ou fait socioéconomique ?. In: Économie rurale. N¹35, 1980. pp. 44-49;

doi : https://doi.org/10.3406/ecoru.1980.2686

https://www.persee.fr/doc/ecoru_0013-0559_1980_num_135_1_2686

Fichier pdf généré le 08/05/2018 


\title{
LA MIGRATION DES AGRICULTEURS FRANGAIS OUTRE-ATLANTIQUE
}

\author{
fait anecdotique, ou fait socio-économique?
}

\author{
Jean-Paul CHARVET \\ Maitre Assistant PARIS $X\left(^{*}\right)$
}

\author{
Violette REY \\ Chargée de Recherche CNRS (*)
}

Cet article cerne le profil d'une nouvelle migration d'agriculteurs qui s'est amorcée en direction de l'Amérique à partir des années 1973-75. Ne touchant que de faibles effectifs, peu organisé, sans institution de soutien, le mouvement est difficile à connaître et n'est pas spécifique aux agriculteurs français ; il recherche des terres disponibles et des structures économiques agricoles plus libérales que celles de la France et du Marché Commun.

Les candidats au départ sont originaires des régions de grande culture (Bassin Parisien, Aquitaine). Trois traits caractérisent leur projet : il correspond plus à une attraction du lieu d'arrivée qu'à un refoulement du lieu de départ. II est indissociable d'un projet d'achat de terres; il ne concerne pas les " paysans pauvres ". Le Canada et l'Argentine sont les premiers pays d'accueil ; la réussite y reste incertaine et difficile parce que les installations ont lieu sur des terres marginales à hauts risques et dans des régions rial desservies. S'eftor
ture.

S'efforçant de mettre à profit le décalage des prix fonciers entre l'Europe et l'Amérique, ce mouvement peut sous certaines conditions intégrer de nouveaux espaces à l'agricul-

Depuis 1973 des petites annonces immobilières proposent aux agriculteurs l'achat de terres au Canada, en Argentine, au Brésil. La grande presse se fait volontiers l'écho, titres tapageurs à l'appui, du nouvel attrait qu'exercent les terres américaines. Mis en présence d'un certain nombre de cas de départs au cours d'enquêtes géographiques menées en des régions diverses (Beaùce, Champagne Marnaise, Berry, Suisse Vaudoise...), nous avons cherché à mieux cerner le phénomène afin de tenter d'apprécier la part du fait anecdotique et celle du fait socio-économique. L'entreprise s'est avérée fort difficile, peut-être prématurée.

\section{UNE MIGRATION LIÉE AU MANQUE DE TER- RE ET AUX FLUCTUATIONS ÉCONOMIQUES}

Le manque de terre pour étendre l'exploitation, la recherche de terres pour installer les enfants, l'incertitude qui existe toujours quant au renouvellement d'un bail... le problème foncier est devenu le problème numéro UN des agriculteurs français et européens. La diminution du nombre des exploitations et des actifs agricoles, accentuée depuis les années 60 , n'a paradoxalement pas atténué « la faim de terres ».
Le rythme d'accroissement du prix des terres agricoles a été supérieur à celui du cours de l'or jusqu'au début de 1979 (1).

L'hectare de terre labourable se négocie actuellement en Beauce autour de $40000 \mathrm{~F}$ et le pas de porte du fermier locataire autour de $10000 \mathrm{~F}$; ces ordres de grandeur sont valables pour l'ensemble des régions de « grande culture » du Bassin Parisien, tout en restant bien inférieurs à ceux observés dans les pays européens voisins (2). Dans ces conditions, les terres américaines, présentées comme disponibles et pas chères, semblent une solution au manque d'espace agricole. Il $\mathrm{y}$ a là un mécanisme né du décalage des prix qui joue actuellement à l'échelle intercontinentale, comme il a joué à l'échelle intra européenne dès les années 50 , lorsque les terres françaises «bon marché » ont attiré (elles attirent encore) certains agriculteurs des pays voisins (BIMA). D'autre part la mobilité et l'émigration agricole ont toujours existé, à l'intérieur même des paysanneries européennes les plus enracinées dans leurs terroirs. Le défrichement et la mise en culture des terres des pays neufs, celle liée à la colonisation d'Afrique du Nord à partir de la seconde moitié du $19^{\mathrm{e}}$ siècle témoignent

(") Membres de l'ézuipe de Géographie Rurale dirigée par Madame BONNAMOUR LA 142, Université de Pais. CNRS. 191, rue Saint-Jacques, Paris 5e. Les rétlexions de cet article n'engagent que leurs auteurs, qui remercient les responsables du CDIR et de la FARME pour leur accueil bienveillant.

(1) Sur la base de 100 en '1950, la terre atteint 250,305, 330, et l'or 52, 144, 150 les années 1965, 1975 et 1978 (SCEES-INSEE).

(2) En 1975, par rapport à la valeur moyenne de la terre agricole trançaise, la terre agricole du Royaumg-Uni coútait environ $25 \%$ de plus, celle des Pays-Bas et du Danemark $55 \%$, celle de RFA pratiquement $100 \%$ et celle de Belgique $225 \%$ de plus. Ct. A. BRUN (1979) "Propriété foncière et exploitation agricole, contradictions et conflits ". Economie Rurale, $n^{\circ} 131, p .29$. 
pour une grande part de ce déplacement d'agriculteurs partis pour rester agriculteurs. Dans la décennie 50, quelques migrations agricoles européennes se sont poursuivies en direction de l'Amérique Latine, alimentée surtout par les séquelles de la Seconde Guerre Mondiale (migrants polonais), par les surplus démographiques de certaines campagnes méditerranéennes (métayers italiens) et par le mouvement de décolonisation du Maghreb (colonies de Pieds Noirds du Maroc) (Wcizman, 1977). Au cours des années 60, les migrations d'agriculteurs européens en direction d'autres continents se sont pratiquement interrompues. Durant cette période, les taux de croissance économique élevés ont offert à la grande majorité des agriculteurs des perspectives d'avenir suffisantes dans leurs pays, soit dans, soit hors de l'agriculture. De plus, sur le marché mondial, les cours des produits agricoles ont stagné jusqu'en 1973 à des niveaux très bas, enregistrant même une perte de valeur en monnaie constante (Charvet, 1978), ce qui incitait peu à quitter l'Europe où des prix agricoles étaient garantis à des niveaux nettement supérieurs. C'est alors au moment où les prix agricoles s'envolent sur le marché mondial, en 1973-75, que s'amorce une sensibilisation au départ vers les terres lointaines.

\section{UN MOUVEMENT DIFFICILE A CONNAITRE}

La difficulté de s'informer, sur les lieux de départ et d'arrivée, se pose aussi bien aux candidats à l'émigration qu'aux observateurs, quoique dans des perspectives différentes. Connaître les possibilités et les conditions d'installation (règlements sur les droits d'activité, de propriété, de circulation des capitaux...), évaluer les flux de personnes et de capitaux, localiser les zones d'implantation sont autant d'éléments que tous souhaiteraient cerner avec plus de précision.

En France une difficulté supplémentaire d'information provient de la grande réserve manifestée à l'égard du mouvement migratoire D'une part, l'écho favorable rencontré par les courants de pensées « vivre au pays » et le jugement critique porté par les analystes des migrations qui considèrent celles-ci comme des processus dissymétriques et inégalitaires (Tapinos, 1974) jouent dans ce sens. D'autre part, on ressent auprès des organisations professionnelles et des responsables agricoles une méfiance, en partie justifiée par les incertitudes et les risques inhérents à ce genre d'opération, en partie soustendue par une politique davantage orientée vers l'action économique et sociale à l'intérieur du cadre national et communautaire.

Les renseignements se trouvent dispersés dans plusieurs directions, aussi bien en France qu'à l'étranger : organismes spécialisés, agences immobilières et banques, presse.

En France. - En 1977 est créée la FARME, Fédération des Associations de Ruraux migrants à l'Étranger, qui installe son siège au CDIR (3), lui-même centre de documentation spécialisé dans l'organisation des stages sur les exploitations françaises ou étrangères et ayant de ce fait une vocation de contact et d'échanges. La FARME reprend les activités d'une association antérieure (Intercampos), animée par des rapatriés installés en Argentine et dans le Sud-Ouest Aquitain. Un tel mécanisme de création par les intéressés de petites associations d'information, puis de regroupement dans des associations plus vastes, reproduit à cinquante ans de distance celui qui se développe dans la France de l'Ouest avec les syndicats locaux de migrations rurales intérieures, tardivement coordonnées par l'ANMER (4). La disparition de l'ANMER, dont les activités ont été reprises par le CNASEA (4), dernière étape de l'institutionnalisation des migrations intérieures, a paradoxalement laissé un vide de fait face à la dimension internationale du mouvement migratoire renaissant. Que la FARME se rattache à une association antérieure de rapatriés, que l'actuel Président de la FARME ait été Président de l'ANMER autrefois, sont révélateurs d'une certaine filiation entre les mouvements migratoires, au niveau des personnes comme au niveau d'un certain style d'action (associations autonomes). La FARME et le CDIR renseignent, organisent des réunions et des voyages d'études, publient un bulletin " Terre des Pionniers " rassemblant des informations technico-économiques et des témoignages d'agriculteurs européens déjà installés.

Les agences privées, soit directement, soit par leurs annonces dans la presse agricole, fournissent des informations lacunaires et dispersées, qu'il convient de confronter aux rares informations officielles. Quant à l'information bancaire, elle est soit très personnalisés pour un client, soit trop générale pour fournir la base d'une étude d'ensemble. Chaque banque a son réseau; parmi les banques nationalisées, le Crédit Lyonnais parait s'être le plus ouvert à cette question. La Banque de France, dans son rapport annuel sur «les mouvements de capitaux à long terme entre la France et l'extérieur ", publie le montant des investissements français à l'étranger dans le domaine agricole mais sans distinguer ce qui concerne l'installation foncière du reste (5).

A l'étranger - Des agences privées très bien organisées existent à notre connaissance en Hollande, Belgique, Allemagne et Suisse (avec publications régulières de bulletins d'information...). Un organisme international, le CIME, Comité Intergouvernemental pour les Migrations Européennes, ayant son siège à Genève, assure la coordination des émigrations avec le souci officiel de favoriser les transferts de technologie. Les flux contemporains d'agriculteurs migrants ne relevant que d'initiatives individuelles passent peu par ses services; cependant son rôle s'accroît avec la création à Viedma en Argentine d'un centre d'accueil et de formation pour les agriculteurs immigrés (1979) (6)

\section{DES CANDIDATS AU DÉPART DE DIVERSES ORIGINES}

La répartition géographique des candidats éventuels au départ comparée à celle des agriculteurs qui ont migré en France entre 1950 et 1965, met en valeur l'originalité du mouvement actuel par rapport aux déplacements antérieurs.

Les cartes ont été établies à partir de la documentation du CDIR (demandeurs d'information, environ 500 cas enregistrés; partici-

(3) Le CDIR, Centre de Documentation et d'Information Rurales, 92, rue du Dessous-des-Berges, 75013 PARIS, est une association sans but lucratif, constituée par une douzaine d'organisations professionnelles et rurales, ayant de multiples activités de diffusion d'information. Outre la revue "Terres des Pionnés ", il diffuse un journal de liaison, la " Lettre aux transhumants ".

(4) ANMER : Association Nationale pour les Migrations et les Etablissements Ruraux

CNASEA : Centre National pour l'Aménagement des Structures des Exploitations Agricoles pants aux réunions et adhérents ayant cotisé, environ 170 cas), elles sous-estiment donc la demande réelle d'information, car la FARME est inégalement connue et les réunions en province encore rares.

Trois sortes de régions à forte demande apparaissent (cf. carte) :

- les régions de «grande culture » céréalière du Centre et plus encore du Nord du Bassin Parisien,

- l'ensemble aquitain, de la Dordogne au Gers,

(5) En 1977, les investissements directs, français à l'étranger, dans le dosmaine agricole, se sont élevés à 68 millions de francs, dont 29 aux US A, 17 ạu Canada, 6 en Amérique 1 atine

(6) La France sest retirée de cet organisme (qui regroupe 32 gouvernements), ce qui ne facilite pas la tâche des agriculteurs francais migrants et des responsables de leurs associations. 


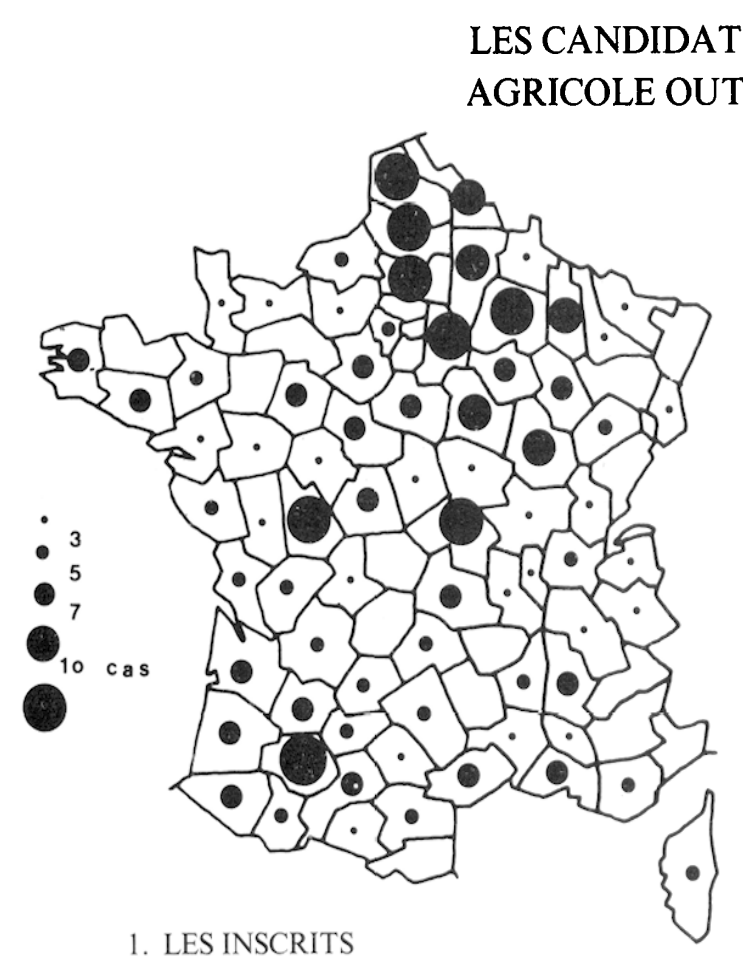

\section{S LA MIGRATION \\ AGRICOLE OUTRE ATLANTIQUE}

1. LES INSCRITS

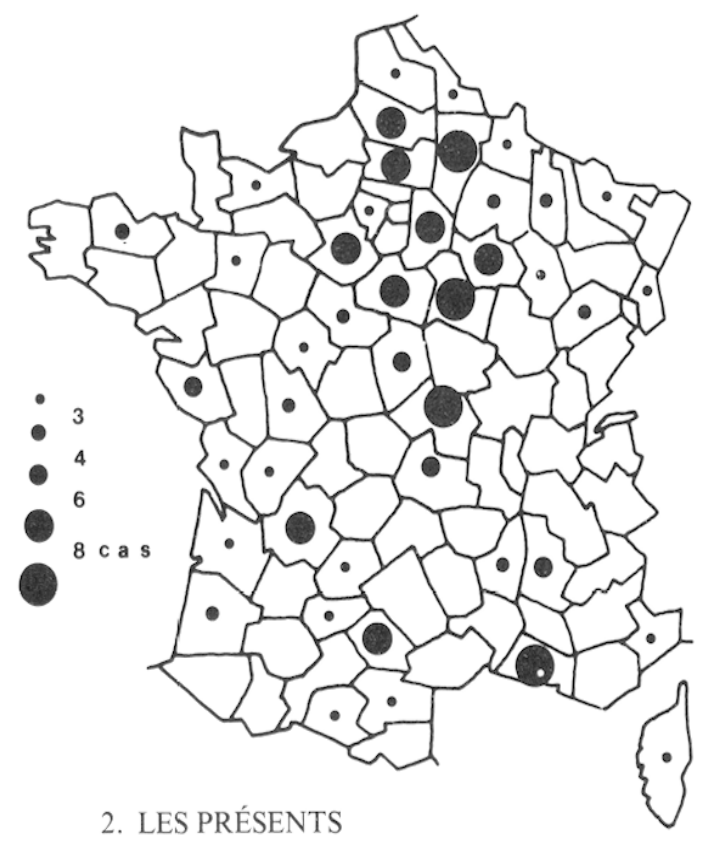

3. LES ABONNÉS
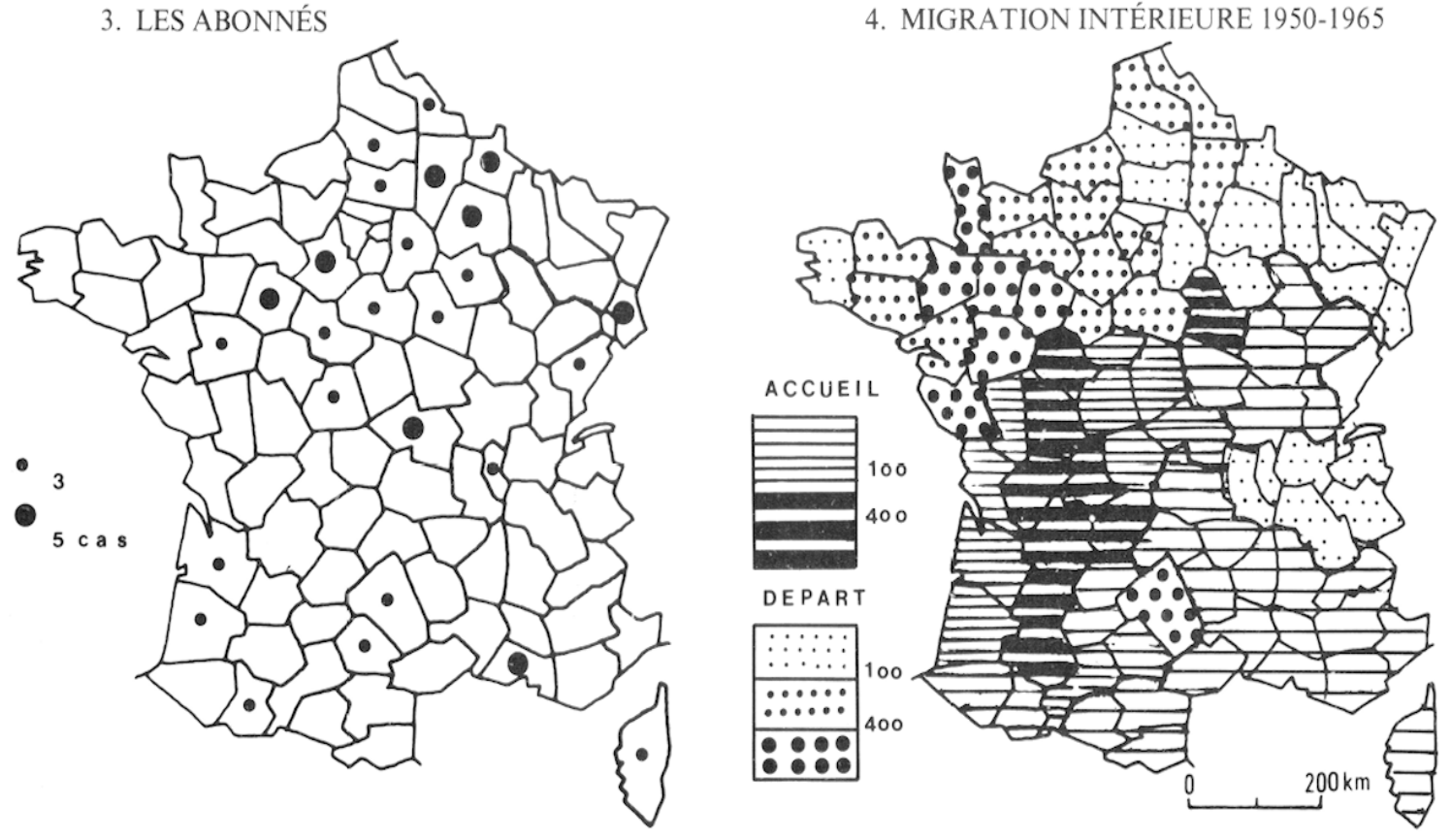

CHARVET. REY 1979

inscrits à la F.A.R.M.E., présents aux réunions, abonnés à la revue.

Cartes élaborées à partir de la documentation aimablement communiquée par le C.D.I.R. 
- des régions dispersées sur le reste du territoire français, Allier, moyenne et basse vallée du Rhône, zone du Poitou-Charente. Ces deux derniers ensembles, qui correspondent aux départements d'accueil de la migration précédente, ont reçu de forts contingents de migrants métropolitains et rapatriés d'Afrique du Nord. Si migration antérieure et migration actuelle conservent des liens dans certains cas, elles ne relèvent néanmoins plus des mêmes contraintes spatiales sur le lieu de départ.Jusqu'en 1960, les fortes densités rurales et les excédents agricoles de l'Ouest avaient provoqué les départs ; de nos jours la mobilité apparaît dans des régions de basses densités, où le rapport Homme/Terre est pourtant bien plus favorable (sauf les régions de montagne, qui n'apparaissent pas pourvoyeuses de candidats potentiels à l'émigration). En d'autres termes se détachent préférentiellement les régions de culture par rapport aux régions d'élevage.

Agriculteurs célibataires et encore jeunes, agriculteurs pères de famille ayant la cinquantaine, sont les deux principaux groupes intéressés tandis que les exploitants des classes d'âge intermédiaires sont au contraire sous-représentés.

Un certain contexte socio-familial individualise ces candidats potentiels au départ : il s'agit d'une tradition familiale dont «la vocation terrienne » se réalise depuis plusieurs générations par la migration. Ce phénomène, déjà observé au niveau de la migration interrégionale française (Rey, 1977) se poursuit donc dans une perspective internationale.

Le système de production pratiqué au départ, le savoir-faire acquis sur l'exploitation antérieure s'avèrent orienter les curiosités et sélectionner l'intérêt porté aux différents pays d'accueil ; tandis que les éleveurs laitiers regardent plutôt vers le Québec, les céréaliculteurs du Bassin Parisien (ou Aquitain) s'intéressent aux terres à blé du Manitoba ou de la Pampa et les spécialistes de cultures maraîchères et fruitières aux périmètres d'irrigation du Piémont des Andes (région du Tucuman).

Sous la diversité des cas particuliers, trois sortes d'occasion provoquent plus fréquemment une sensibilisation à l'émigration éventuelle. L'expropriation pour cause d'utilité publique et l'éviction par reprise destructurent et affaiblissent l'outil de travail, mais laissent au chef d'exploitation un volume de capitaux à rapidement ré-utiliser dans la conjoncture monétaire actuelle : l'investissement en terre à l'étranger peut alors être une solution. (La région parisienne fournit plusieurs exemples de ce type). Les calamités agricoles, surtout si elles se répètent plusieurs années consécutives et si elles affectent des exploitations en situation financière précaire peuvent susciter un brusque intérêt pour le départ vers d'autres horizons ; l'Allier, le Gers après les inondations de 1977 en donnent des exemples. Enfin le réseau des relations habituelles demeure le canal privilégié de diffusion de l'information.

Les agriculteurs intéressés par un départ éventuel appartiennent à quatre types :

- L'entrepreneur, l'homme qui a, sinon le goût de l'aventure, du moins l'esprit du pionnier et du bâtisseur. Il cherche hors d'Europe les grands espaces où agir, dégagé des multiples carcans réglementaires qui enserrent l'agriculture française (la législation sur les cumuls est souvent visée par ses propos.

- L'investisseur qui, d'une façon plus ou moins explicite considère qu'il est sage de ne pas «mettre tous ses œufs dans le même panier » et envisage de mettre à l'abri sur un autre continent une part de son patrimoine. Le placement en terre, vers lequel se convertissent également nombre d'autres investisseurs (banquiers) (7), apparaît une excellente mise d'autant que la terre est le bien qu'il connaît le mieux.

- Le jeune qui veut démarrer dans le métier, mais ne peut rester sur l'exploitation des parents (trop petite ou famille nombreuse), tout en sachant qu'il pourra compter sur l'aide financière de ces derniers.

- Enfin, l'agriculteur de la quarantaine qui par des circonstances diverses se trouve en difficulté et qui voit dans le départ la possibilité d'une deuxième chance.

\section{Deux réflexions s'imposent ici :}

«les paysans pauvres" selon l'expression de certains analystes, "laissés pour compte" de l'évolution agricole contemporaine, ne participent pas au mouvement d'intérêt suscité par l'émigration; quelle que soit la situation financière au départ, le projet d'émigration est indissociable d'un projet d'achat de terre. Moins refoulés du lieu d'origine qu'attirés par les possibilités à l'arrivée, ces candidats ont l'initiative de leur projet et comptent mettre à profit la rente différentielle de situation, née du décalage entre les prix européens et les prix américains de la terre.

\section{DES REGIONS D'ACCUEIL OU LA REUSSITE EST INCERTAINE}

Combien sont-ils à s'être installés OutreAtlantique? Ont-ils dépassé la centaine depuis $1972-73$ ? Cela n'est pas certain. Un double décalage très marqué existe entre ceux qui se sont informés en France et ceux qui ont effectué un voyage d'études, comme entre ces derniers et ceux qui ont acquis de la terre et l'exploitent. En outre le mouvement ne peut être que marginal, compte-tenu du volume de terres effectivement disponibles.

Comparativement à l'Afriquc ou à l'Australie, l'Amérique jouit d'une très nette préférence. Elle pré- sente encore une certaine disponibilité de terres (Amérique du Sud). Proche culturellement de l'Europe, elle l'est aussi en distance-temps et argent (Amérique du Nord), et ces proximités ont un impact psychologique non négligeable dans l'élaboration des projets. Son image de marque associe encore la relative stabilité des régimes politiques et la liberté d'entreprise. Enfin certains pays maintiennent une politique d'accueil ; appréciant une certaine capacité d'investissement et

(7) Ct. le mouvement des investisseurs étrangers sur les terres agricoles des Etats-Unis. 
plus encore un savoir-faire technologique, leurs gouvernements cherchent à sélectionner des migrants « de qualité » : Argentine et Canada sont les deux principaux « réceptacles » de ce mouvement migratoire agricole, avec des conditions d'accueil et des possibilités agricoles différentes.

En Argentine, le réseau des infrastructures de transport, de commercialisation (stockage) et de services devient très lâche dès que l'on s'éloigne de la Pampa " humide» (région de Buenos-Aires). Le gouvernement argentin peut accorder certaines primes de défrichement et de mise en valeur, mais il n'y a guère de possibilités réelles de recours au marché local des capitaux pour des achats de terres: les prêts les plus longs ne dépassent pas deux à trois ans et présentent des taux d'intérêt très élevés, cette situation étant la conséquence d'une inflation très forte. Dans l'attrait supérieur exercé par l'Argentine par rapport au Brésil, l'image de terres «tempérées " dont les possibilités agricoles sont proches de celles de l'Europe Occidentale, s'ajoute à une politique d'accueil plus active.

Au Canada, le contexte social et les conditions d'activités sont plus proches de ceux de l'Europe: la commercialisation des produits agricoles est mieux assurée grâce à des structures techniques et financières plus élaborées; l'obtention de prêts d'installation et d'équipement est relativement aisée (8) ; enfin l'usage de la langue française et la filière bien établie des stages agricoles ne manquent pas de faciliter les contacts et la première insertion.

Ces différences se retrouvent au niveau des profils et de l'origine des migrants déjà installés.

Les cas d'établissement semblent plus nombreux au Canada qu'en Argentine malgré des prix moyens plus élevés.

L'analyse d'une centaine d'offres de terres provenant de deux importantes agences immobilières, l'une canadienne, l'autre argentine, montre que $75 \%$ des propositions se font sur la base de prix supérieurs à 800 dollars au Canada alors que c'est l'inverse en Argentine (9). De ce fait, ce ne sont pas les mêmes migrants qui s'installent en Amérique du Nord et du Sud : les premiers préferent s'établir sur des exploitations déjà existantes, pourvues de bâtiments et relativement équipées plutôt que de courir tous les risques d'un premier défrichement sur des parcelles assez souvent mal délimitées (10).

Il existe certes à proximité de Buenos-Aires comme de Winnipeg d'excellentes terres de culture desservies par un réseau dense d'infrastructures de transport et de services, mais leurs prix se rapprochent de ceux pratiqués dans le Bassin Parisien. Toutefois les terres les plus aisément disponibles et les plus attractives par leurs prix sont en majorité et à des degrés divers, des terres marginales situées sur des franges climatiques et correspondant à des sols mal connus. Donc des terres à hauts risques. Il s'agit des confins de la Pampa humide et de la Pampa sèche, des terres subtropicales de la province de Salta (au Nord de l'Argentine) encore souvent couvertes de forêt, ou des secteurs de bordure de la Prairie Canadienne où la durée de la période végétative devient particulièrement réduite. Dans la plupart des cas, l'utilisation d'un matériel puissant, permettant de travailler

(8) Pour l'achat de terre, les prêts peuvent être accordés sur une durée de 15 ans : pour les achats de matériels d'équipement, sur une durée de 5 ans.

(9) Pour le Canada: ROBIDOUX REALTY (Winnipeg, Manitoba). Pour l'Argentine :AGRO PALERMO S.A. (Buenos-Aires).

(10) Sans parler des problèmes de clôtures et de titres de propriété plus ou moins bien établis.

(11) Cf. Walter M. KOLLMORGEN et George F. JENKS. - " Suitcase farming in Sully County, South Dakota .. Annals of the Association of American Geographers, Vol. 48 , Mars $1958, N^{\circ} 1, p .27-40$. - Lesitio HEWES. - The suitcase farming frontier, a study in the historical geography of the Central Great Plains $n$. University of Nebraska, 1973. vite, s'avère indispensable. Les capacités réelles des sols sont difficiles à cerner de facon rapide (comportement de l'eau dans les sols, composition en oligo-éléments, réaction aux engrais). Dans les zones semi-arides chaudes s'ajoutent les risques de salinisation, sans parler de dangers d'érosion des sols caractéristiques de bien des régions restées jusqu'à notre époque faiblement mises en faleur.

Donc à l'échelle internationale comme à l'échelle française, les migrants se trouvent souvent dirigés vers la mise en valeur de terres marginales. Ainsi s'éclaire mieux leur tendance à l'instabilité et à la mobilité. A l'intérieur du groupe des migrants, ceux qui disposent des bases de départ les plus solides savent et peuvent choisir l'aventure minimale. Certains parmi eux n'opèrent qu'une extension et conservent leur unité de production française : ils peuvent utiliser ainsi les inversions de saisons liées au changement d'hémisphère ou au moins les décalages de périodes de gros travaux existant entre des régions de climats fort différents. « Det farmers » selon une expression de journaliste, ils reprennent une tradition de mobilité en partie comparable à celle des " suitcase-farmers " et étatsuniens (11).

Le phénomène de la migration agricole OutreAtlantique apparaît donc comme un phénomène «à la marge " s'articulant sur deux ordres de fonctionnement économique : celui des agricultures de l'Europe de l'Ouest, à l'étroit dans leurs espaces de production, leurs structures et leurs réglementations face aux agricultures du Nouveau Monde qui bénéficient souvent d'une liberté d'action beaucoup plus importante liée à la relative souplesse de leur organisation et à la possibilité d'utiliser ou non un volant de terres disponibles (12) ; celui du mouvement plus général d'investissement en direction du foncier choisi par les porteurs de capitaux, agriculteurs ou non, en temps de crise économique (13).

Malgré sa marginalité, ce type de mouvement migratoire peut contribuer, à partir du moment où il dispose de moyens suffisants, à régénérer, voire à intégrer progressivement de nouveaux espaces à l'agriculture. En favorisant la comparaison entre des espaces très éloignés et l'ouverture à de nouvelles techniques comme à de nouveaux horizons économiques et culturels, il laisse présager des retombées bénéfiques dans des régions et pays de départ. C'est le calcul que semblent avoir fait certains de nos voisins (14).
(12) CHOMINOT A. - Quelques tendances nouvelles de l'agriculture des Etats-Unis * Annales de Géographie $N^{*} 467,1976$, p. 34-47.

(13) Dès 1975, aux Etats-Unis, la chronique journalistique s'est faite l'écho de l'inquétude des fermiers devant la progression des achats de terres agricoles par les investisseurs étrangers, surtout europeeens. Plusieurs HOMA) ont pris des mesures très limitatives à leur encontre. L'USDA publie un rapport annuel sur ce mouve-
Hom ment depuis 1976.

(14) Les auteurs considèrent cet article comme une contribution préliminaire à l'étude de ce mouvement, in seréé dans des recherches plus générales sur la consommation d'espace par l'agriculture. lls recevront volontiers toutes les informations et les remarques susceptibles d'élargir le champ de leur réflexion. 


\section{BIBLIOGRAPHIE}

CHARVET J.-P. (1977). - La flexibilité de la production de blé aux Etats-Unis. Bulletin de l'Association des Géographes Français, $n^{\circ} 448$, p. $331-342$.

REY V. (1977). - Le thème de la migration agricole en France. Cahiers de Fontenay, n` 7, p. 65-91.

REY V. (1977). - «Les agriculteurs migrants, le cas du Cher». Études Rurales, $n^{\circ} 68$, p. 55-91.

REY V. (1979). - Want of farming space and migrations of farm people, a consequence of modern agriculture in «the affect of modern agriculture upon the rural transformations ». Pergamon press, New York.

TAPINOS (1974). - «L'économie des migrations internationales ». A. Colin, $290 \mathrm{p}$.

WEIZMAN H. (1977). - « La migration rurale vers l'Amérique Latine, les leçons d'une expérience récente». Troisième séminaire sur l'adaptation et l'intégration des immigrants permanents, CIME, Genève (16, avenue J. Tremblay), $33 \mathrm{p}$.

\section{ARTICLES DE PRESSE}

- «Treize alsaciens dans la Pampa». Nouvel Observateur, 5/3/ 1979.

- «Deux agriculteurs français au Brésil : l'aventure, ça existe encore " Agrisept, 8/6/1979.

- "The foreign land-grab scare : xenophobic over reaction to a bit of buying in the farm belt ». Time, 8/01/1979.

\section{Sur les investissements fonciers étrangers}

- En France :

- Ministère de l'Agriculture : «Installations et acquisitions des étrangers en France ». Rapports 1973-74-75-76 du BIMA.

- Aux Etats-Unis :

- DUNCAN M. (1977). - «Farm real estate: who buys and how?" Monthly review of Federal Reserve, Bank of KANSAS CITY, $25 \mathrm{p}$.

- WUNDERLICH G. (1976). - «Foreign investment in US real estate». USDA Agricultural information bulletin $\mathrm{N}^{\circ} 400,28 \mathrm{p}$.
- Le Monde du Dimanche (1979). - En 1979, moins de 0,5\% des terres agricoles privées US appartiennent à des étrangers, soit environs 1,6 millions d'hectares, dont 367000 pour les britanniques, 160000 pour les Luxembourgeois, 142000 pour les Allemands. Le Monde du Dimanche, 14/10/1979.

Annexe 1 : Quelques exemples d'offres proposées par AGRO PALERMO S.A. (Buenos Aires) début 1979:

1. Région de SANTIAGO DEL ESTERO (province de SALTA, au nord du pays):

- 2450 ha à $2 \mathrm{kms}$ de la route 34 , terres pouvant convenir à la culture du soja et du sorho plus un secteur montagneux boisé. Sources.

- 258000 dollars soit 105 dollars/ha.

2. Région de CORRIENTES (vallée du Parana)

- 750 ha à $6 \mathrm{kms}$ de la route 5 , terres pouvant convenir à la culture et à l'élevage.

- 105300 dollars soit 140 dollars/ha.

3. Région de CORRIENTES:

-10219 ha à $5 \mathrm{kms}$ de la route 12 , déjà 300 ha reboisés; terres pouvant convenir à l'élevage, aux cultures ou à l'horticulture.

-3100000 dollars soit 303 dollars/ha.

4. Région de SANTA FE :

- 573 ha le long de la route 33 , matériel ; bâtiments en bon état ; terres franches (terres à

-1031000 dollars soit 1800 dollars/ha.

Annexe 2 : Quelques exemples d'offres proposées par ROBIDOUX REALTY (Winnipeg, Manitoba, Canada) en 1978 :

1. Ferme céréalière de la région de NEEPAWA (centre sud du Manitoba):

- 640 acres (256 ha), sols de classe 4 et 5 (sols médiocres à très médiocres), pas de bâti-

111000 dollars soit 434 dollars $/ \mathrm{ha}$

2. Ferme céréaiière de ia région de SELKiRK (esl du Manitoba, au nord de Winnipeg) :

- 960 acres ( 384 ha), sols de classe 4 (sols médiocres), pas de bâtiments.

600 dollars soit 525 dollars/ha.

3. Ferme céréalière de la région de MONT RIDING (centre ouest du Manitoba) :

- 2240 acres ( $896 \mathrm{ha}$ ), sols de classe 2 (assez bons sols, assez profonds et retenant l'eau de facon convenable), maison d'habitation, cellules à grain.

- 900000 dollars soit 1005 dollars/ha.

4. Ferme céréalière de la région de BRANDON (centre sud du Manitoba) :

- 960 acres ( $384 \mathrm{ha}$ ), sols de classe 2 (assez bons sols), bonne maison d'habitation, hanar pour le matériel, cellules à grain.

- 604800 dollars soit 1575 dollars/ha.

5. Ferme céréalière et à légumes de la région de WINNIPEG (vallée de la Red River):

- 640 acres ( $256 \mathrm{ha}$ ), sols : $70 \%$ de classe 1 (bons sols, profonds, à la fois bien drainés et retenant bien l'eau, faciles à travailler) et $30 \%$ de classe 2 (assez bons sols). Très bonne maison d'habitation et bons bâtiments de ferme.

-675000 dollars soit 2637 dollars/ha. 